\title{
Argument \& Computation Community Resources (ACCR) corner
}

\author{
Pietro Baroni and Bart Verheij
}

With this editorial, we introduce the Argument \& Computation Community Resources (ACCR) corner of the journal. The corner's goal is to provide useful and citable archival references for valuable community resources whose dissemination is typically limited to semi-formal venues (like web sites) as they do not fit the conventional notion of journal article.

The notion of community resource is inherently open and includes software tools, benchmarks, text corpora, etc. In principle anything satisfying the requirements specified below is eligible to be included in the ACCR corner. We aim to publish short articles describing resources featuring the following properties:

- Relevance: the resource should address some general need relevant to a significant investigation topic inside the Argument \& Computation research community.

- Utility: there should be empirical (e.g., based on use cases) or theoretical (e.g., based on formal properties) evidence of the practical utility of the resource.

- Reusability: the resource should be reusable with a reasonable effort by other researchers investigating the same topic.

- Availability: the resource should be publicly available.

The typical expected contribution to the ACCR corner is a short (indicatively from 4 to 6 pages) but selfcontained description of a resource. The description should address (synthetically) at least the following three aspects (which may correspond to the article sections).

1. Introduction and context: a description of the typology of the resource, of the investigation topic to which it is meant to contribute, and of its potential utility to other researchers.

2. Resource description: a detailed description of the resource, including an explanation of its relevance and added value/originality with respect to other existing resources.

3. Resource use: a description of past uses of the resource (e.g., in a competition or published research), demonstrating its utility, together with short instructions for prospective users (possibly referring to external documentation if needed) and indications on the availability of the resource.

Also a report on a resource-based event such as a past competition can be considered for the ACCR corner. Contributions to the ACCR corner will undergo a review process, expected to be faster than usual due to their synthetic nature. Extensive descriptions significantly exceeding the suggested size will not be acceptable for the ACCR corner and should be considered as regular submissions.

Pietro Baroni and Bart Verheij

July 2019

1946-2166/19/\$35.00 @ 2019 - IOS Press and the authors.

This article is published online with Open Access and distributed under the terms of the Creative Commons Attribution Non-Commercial License (CC BY-NC 4.0). 